\title{
The effect of leaf presence on the rooting of stem cutting of bitter melon and on changes in polyamine levels
}

\author{
Nasir S. A. Malik ${ }^{1 * \#}$, Jose L. Perez ${ }^{1}$, Madhurababu Kunta ${ }^{1,2}$ \\ ${ }^{1}$ United States Department of Agriculture, Agricultural Research Service, Weslaco, USA; \\ \#Corresponding Author: Nasir.malik@ars.usda.gov \\ ${ }^{2}$ Texas A \& M University-Kingsville, Citrus Center, Weslaco, USA
}

Received 7 August 2012; revised 10 September 2012; accepted 7 October 2012

\begin{abstract}
The study was conducted to investigate the optimal hormone treatment for rooting in bitter melon and the effect of defoliation on rooting and polyamine levels. Commercial preparation (diluted 1:10 and 1:20) gave extensive rooting within five days after treatment. The presence of leaf with the stem cutting was necessary for optimal rooting as defoliation drastically reduced rooting even when the rooting time was extended from 5 to 10 days. The presence of leaf also promoted the levels of putrescine three days after the hormone treatment just at the time of root emergence. Cuttings that did not have leaf did not show an increase in putrescine levels and did not produce roots. Spermidine levels also increased in the cuttings that rooted but there was no change in the levels of spermine in the cuttings that rooted. It appears that putrescine and spermidine play a role in regulating rooting in bitter melon cuttings. The vegetative propagation conditions described show that it is a practically viable technique for small growers to produce large number of plantlets at the farm for selection and cultivation.
\end{abstract}

Keywords: Momordica charantia; Polyamines; Rooting; Defoliation; Propagation

\section{INTRODUCTION}

Bitter melon (Memoridica charantia L.) is also known as bitter gourd and is grown in tropical and subtropical climates of India, Pakistan, China and other South Asian countries as vegetable for food and medicinal purposes

\footnotetext{
*Current address: USDA-ARS Eastern Regional Research Center, 600 East Mermaid Lane, Wyndmoor, PA 19038.
}

[1-3]. It has a bitter taste but is still considered a culinary delicacy in Indian-subcontinent for its special taste and high nutritive value, and therefore, it is generally sold as a premium priced vegetable [1-4].

In addition to being a high valued vegetable, bitter melon is used for numerous medicinal purposes and is renowned for its antidiabetic or glucose lowering effects [5-7]. Various fraction of bitter melon extracts have been shown to increase glucose uptake and improve insulin resistance which are important factors for controlling Type-2 diabetes $[8,9]$. Although it is most widely used to control diabetes, bitter melons powders and extracts from fruits and seeds have also been used to improve digestion, manage HDL-cholesterol levels, treatments for ulcers, hypertension, microbial and viral infections, intestinal inflammation, and obesity [3,5,7,10-16].

Owing to its importance as prized vegetable and effective herbal medicine, researchers have been interested to improve its propagation, production, and processing techniques. For example, molecular techniques have been developed to identify different cultivars and then in vitro cultivation techniques were developed for mass propagation of selected cultivars and to use these methods for genetic transformation $[1,17,18]$. Grafting techniques have been developed to produce cultivars tolerant to flooding, and hormonal roles have been tested for manipulating budding and flowering in bitter melon [1921].

Propagation through tissue culture has the advantage of mass production and the ability to produce possible transformations but the technique requires sophisticated laboratory facilities. This study was conducted to develop optimum conditions for rooting bitter melon cuttings for mass production of plantlets with minimum facilities at the farm. Our preliminary experiments indicated that presence of a leaf on the cuttings may be an important factor in rooting success. Therefore, experiments were conducted to evaluate the role of leaf on 
rooting. In addition, changes in polyamine levels in the rooting zone of the cutting were also measured because polyamine have previously been implicated in the rooting phenomenon $[22,23]$. The results of these studies are described here.

\section{MATERIALS AND METHODS}

\subsection{Plant Material and Rooting Experiments}

Bitter melons grown in field at USDA-ARS facility in Weslaco were used as a source for obtaining cuttings for the rooting experiments in this study. Cuttings were dipped in appropriate hormone concentration in the field (see below) and the cuttings were placed in locally designed rooting setups (Figure 1(a)) which were then brought to greenhouse.

The bottom plastic pots $(9 \times 9 \times 9 \mathrm{~cm})$ of the rooting systems were filled with a mixture of perlite:vermiculite (1:1) moistened with deionized water. Typically, a cutting was cut $5 \mathrm{~cm}$ below, and $1 \mathrm{~cm}$ above, a node with a leaf. About $3 \mathrm{~cm}$ of the basal portion of the cutting below the leaf was dipped in appropriate concentration of the hormone and planted in the rooting medium described above. Normally, a $4-5 \mathrm{~cm}$ wide leaf was left attached to each cutting, except for the defoliation experiments. Immediately after planting the cutting ( 2 cuttings per pot), a clear plastic cup $(8.5 \mathrm{~cm}$ diameter and $11 \mathrm{~cm}$ tall $)$ was placed on top of the cuttings. This cup was sprayed with water and then securely placed in the perlite:vermiculite (1:1) mix of bottom plastic pot to maintain high humidity around cutting and the leaf (Figure 1(a)).

\subsection{Preparation and Application of Rooting Hormone}

Commercially available "Liquid Rooting Concentrate" manufactured by DIP'N GROW (Clackamas, Oregon 97015, USA) was used in these studies. The concentrate was diluted as indicated in each treatment before use. The concentrate contained 1\% indole-3-butyric acid and $0.5 \%$ naphthalene acetic acid; and 98.5\% inert ingredients. For comparative studies, a laboratory mixture of the two hormone was prepared from reagent grade compounds supplied by Sigma Chemical Co. The cuttings were dipped for 10 seconds in appropriate dilution before planting in the rooting medium.

Scoring. At various intervals after hormone treatment the cuttings were scored for the extent of rooting by two individuals following the scoring guide shown in Figure 1(b). A unified score was recorded for each cutting; and twenty replicate cuttings were scored for each treatment.

\subsection{Polyamine Analysis}

Polyamine extraction. Polyamines were extracted from

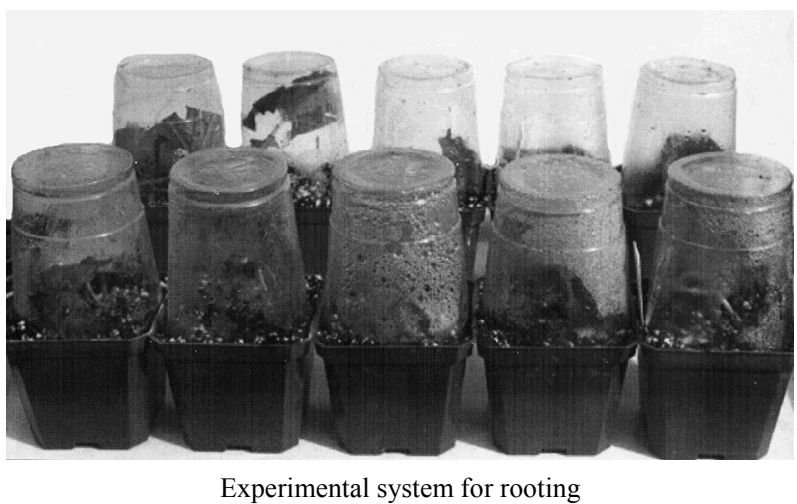

(a)

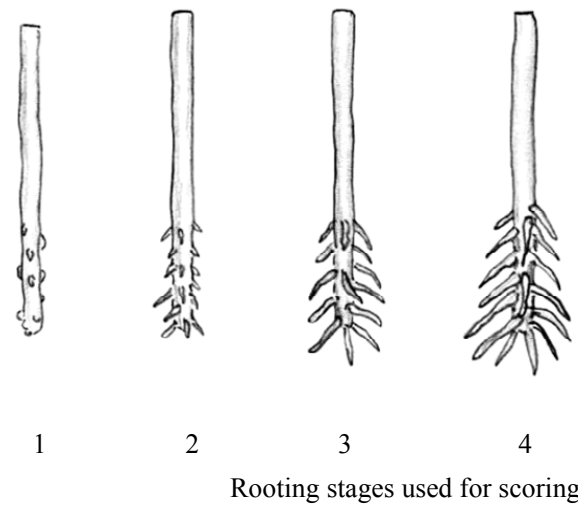

(b)

Figure 1. (a) The design of the rooting units used to induce rooting. The pots were filled with potting mix supplied by SunShine Co. The clear plastic cups were sprayed with distilled water to keep high humidity; (b) The rooting stages that were used as a guide for scoring extent of rooting in response to different treatments.

the lower $3 \mathrm{~cm}$ portions of cuttings three days after hormone treatments. At this point, the cuttings with leaves were swollen and had emerging roots, while cuttings without leaves were devoid of such signs. The portions of root samples were immediately frozen in liquid nitrogen and then pulverized in liquid nitrogen before extraction following our standard method [24]. About $300 \mathrm{mg}$ aliquot of the frozen powdered leaf material was mixed with $8 \mathrm{~mL}$ of $5 \%$ perchloric acid. The mixture was sonicated for 30 seconds and shaken overnight at $4{ }^{\circ} \mathrm{C}$, and then centrifuged at $5000 \mathrm{~g}$ for $45 \mathrm{~min}$. The resulting supernatant liquid was purified by ion exchange chromatography as follows. A slurry of the resin AG50-X8 was poured into a glass column $(9 \mathrm{~mm} \times 100 \mathrm{~mm})$ where the slurry settled to $50 \mathrm{~mm}$ height. Before pouring the resin slurry into column, it was equilibrated for $2 \mathrm{~h}$ with 5 bed volumes of starting buffer consisting of $0.7 \mathrm{M}$ sodium chloride in $0.1 \mathrm{M}$ sodium phosphate (dibasic) solution. The column was first eluted with $10 \mathrm{~mL}$ of starting buffer and then $2 \mathrm{~mL}$ of extract was added at the top of the resin column. After the extract was absorbed in the 
resin, the column was washed with different solvents in the following order: three $\mathrm{mL}$ of starting buffer followed by $2 \mathrm{~mL}$ of MilliQ water and then $3 \mathrm{~mL}$ of $1 \mathrm{M} \mathrm{HCl}$. The polyamine bound to the resin was then eluted with $5 \mathrm{~mL}$ of $6 \mathrm{M} \mathrm{HCl}$ at the rate of $0.5 \mathrm{~mL} /$ minute. The eluents containing polyamines were collected in $2 \mathrm{~mL}$ microfuge tubes and then dried using a speedvac. The dried samples were either derivitized as described below or stored at $-80^{\circ} \mathrm{C}$ until use.

Derivatization of polyamines. The dried samples were reconstituted in $0.2 \mathrm{~mL}$ MilliQ water and combined with $1.2 \mathrm{~mL}$ of dansyl chloride and $0.6 \mathrm{~mL}$ of saturated sodium carbonate solution in a $15 \mathrm{~mL}$ tube. The mixture was vortexed and was heated at $50^{\circ} \mathrm{C}$ for $1 \mathrm{~h}$ in the dark. After allowing the mixture to cool to room temperature, a $0.3 \mathrm{~mL}$ aliquot of proline $(100 \mathrm{mg} / \mathrm{mL}$ of water) was added and allowed to incubate in the dark for $30 \mathrm{~min}$. After centrifugation, $4 \mathrm{~mL}$ of toluene was added to the supernatant liquid and the mixture was vortexed and centrifuged briefly to allow separation of phases. The organic layer was discarded and the aqueous layer containing the derivatized polyamines was dried in a speedvac. The dried sample was reconstituted in $0.2 \mathrm{~mL}$ of methanol. This reconstituted sample was used for HPLC analysis as previously described [24].

High performance liquid chromatography of polyamines. The derivatized polyamines from the extracted tissue were separated using Waters Symmetry C18 (5 $\mu \mathrm{m}$ particle size $)$ column $(3.9 \mathrm{~mm} \times 150 \mathrm{~mm})$ maintained at $25^{\circ} \mathrm{C}$ during chromatographic runs. A $10 \mu \mathrm{L}$ sample was injected into the HPLC for analysis. The column was eluted at a flow rate of $1 \mathrm{~mL} / \mathrm{min}$ with a gradient of solvent system made up of HPLC grade methanol (solvent A, 60\%) and MilliQ water (solvent B, 40\%). The elution was started with solvent mixture comprising of $60 \% \mathrm{~A}$ and $40 \%$ B. The component A was linearly increased up to $95 \%$ in 23 minutes and then maintained isocratically for 7 minutes. The polyamines were detected with a fluorescent detector at $365 \mathrm{~nm}$ wavelength and excitation of $510 \mathrm{~nm}$ wavelength for emission. For quantitative measurement of different polyamines in the extracts, regression curves were developed using known standard compounds.

\section{RESULTS}

The effect of different dilution of the commercial rooting hormone on the extent of rooting of bitter melon cuttings is shown in Figure 2(a). Ten and 20 times dilution of the hormone concentrate significantly increased rooting compared to controls with no hormone treatment or treatment with high concentration of hormone (i.e. 1:5 dilution) (Figure 2(a)). The commercial preparation of rooting hormone seemed as good as or better than the laboratory preparation of the same ingredients at same concentrations (Figure 2(b)).

The presence of leaf with the cutting had profound effect on rooting of the cutting (Figure 3 ). Even when the incubation time to evaluate rooting was extended to 10 days, rooting remained very minimal without leaf (Figure 3).

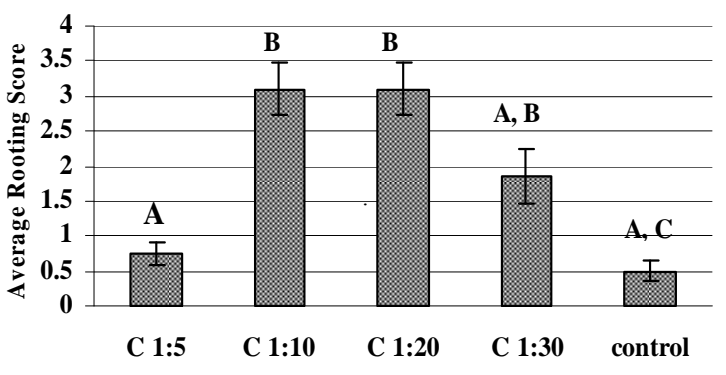

(a)

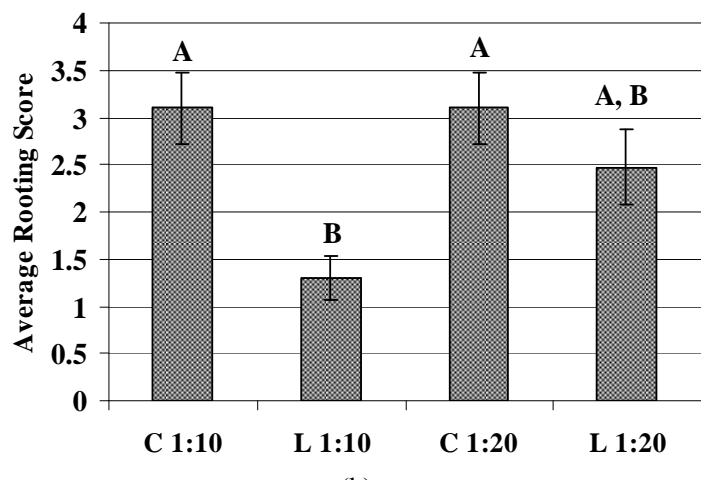

(b)

Figure 2. (a) The effect of various dilutions (1:5 - 1:10, $1: 20,1: 30$ and pure water; i.e., control) of commercial rooting hormone on the extent of rooting in bitter melon cuttings. The cutting was dipped in the hormone dilution for 10 seconds and then incubated for 9 days in the rooting system described in Figure 1; (b) Comparison between commercial (C) and laboratory (L) prepared hormone dilution with same concentrations of active ingredients.

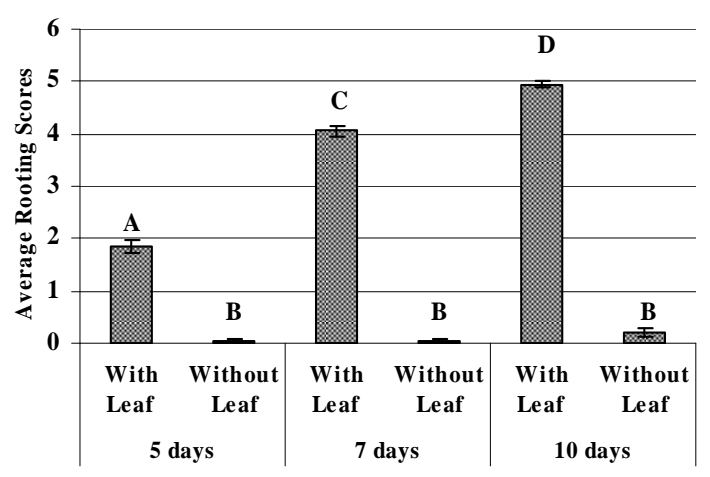

Figure 3. The effect of presence and absence of leaf on the extent of rooting in bitter melon stem cuttings. The cuttings were dipped in commercial hormone (1:20) for 10 seconds and then incubated for 10 days. 
Putrescine levels were significantly increased in cuttings three days after dipping in hormone (commercial diluted 1:20) with the leaves compared to initial levels (Figure 4). On the other hand similarly cut and treated cutting but kept for three day without leaves had significantly lower amounts of putrescine (Figure 4). Spermidine levels also significantly increased in cuttings with leaves three days after hormone treatment compared to initial levels and also compared to levels in similarly treated cuttings but without leaves (Figure 4). There was no significant difference in spermidine levels in defoliated cuttings three days after hormone treatment with the initial levels (Figure 4). Spermine levels did not change significantly in cuttings with or without leaves three days after hormone treatment from the initial levels (Figure 4).

\section{DISCUSSION}

It has been known for a long time that indole butyric acid and naphthalene acetic acid can induce rooting in stem cuttings of various plants although concentration required would be different for different plants [25]. The results from this study, while confirming in bitter melon the effect of rooting hormone, they also indicate the concentration that are most effective in promoting rooting. The studies also provide information that commercial preparation of rooting hormone sold in the market are just as effective as preparation made from pure compounds in the laboratory and this should be valuable information for small to medium scale growers of bitter melons.

Leaf presence may or may not play an important role in rooting of specific plant species [25]. For example, sweet potato cuttings in our hand rooted just fine (unpublished results) but absence of leaves in bitter melon cuttings drastically reduced rooting even with the hormone treatment (Figure 3). This is important information for growers as well as a valuable tool for researcher trying to understand the regulatory mechanism of rooting.

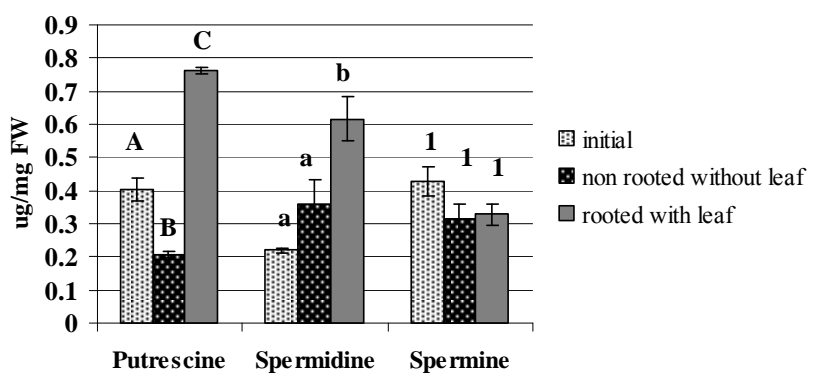

Figure 4. Comparison of polyamine levels in bitter melon cuttings with and without leaves. The cuttings were dipped in commercial rooting hormone (1:20) for 10 seconds and incubated in the rooting medium until the cuttings with leaves showed the emergence of roots (stage 1). The bottom $3 \mathrm{~cm}$ of cuttings were used analyzing polyamine levels.
For this purpose, we looked at changes in polyamine levels in hormone-treated cuttings in the presence and absence of leaves. Polyamines have been implicated in vegetative rooting of stem cuttings in different species [22,23,26-28]. Thus, consistent with previous reports, putrescine levels significantly increased in the bitter melon cuttings that were about to root; i.e., cuttings with one intact leaf and treated with hormone [23,27,28]. The involvement of putrescine levels in rooting of bitter melon stem cutting, as shown in other species, is supported by the fact that the putrescine levels did not increase in defoliated cuttings that did not root [23]. The role of other polyamines such as spermine and spermidine in regulating adventitious root formation is less clear as researchers in 1983 found increased levels of these polyamine in mug bean cutting just before rooting but later study in 2001 found no such increases, while our study with bitter melon shows an increase only in spermidine [27,28].

In general, the results of this study show that leaf presence has a profound effect on the rooting of bitter melon stem cutting. Apparently, leaf presence promotes rooting through increase in the levels of putrescine, and perhaps spermidine, in the rooting zone of the bitter melon stem cutting.

\section{REFERENCES}

[1] M Dey, S.S., Singh, A.K., Chandel, D. and Behera, T.K. (2006) Genetic diversity of bitter gourd (Momoridica charantia L.) genotypes revealed by RAPD markers and agronomic traits. Scientia Horticulturae, 109, 21-28. doi:10.1016/i.scienta.2006.03.006

[2] Al Mansur, M.A.Z., Haque, M.S., Nasiruddin, K.M. and Hossain, M.S. (2009) In vitro propagationof bitter gourd (Momoridica charantia L.) from nodal and root segments. Plant Tissue Culture and Biotechnology, 19, 45-52.

[3] Jadhav, D.B., Visavale, G.L., Sutar, P.P., Annapure, U.S. and Thorat, (2010) Solar cabinet drying of bitter gourd: Optimizing of pretreatment and quality evaluation. International Journal of Food Engineering, 6, 1-18. doi:10.2202/1556-3758.1503

[4] Behera, T.K. (2004) Heterosis in bitter gourd. Journal of New Seeds, 6, 217-222. doi:10.1300/J153v06n02 11

[5] Jayasooriya, A.P., Sakono, M., Yukizaki, C., Kawano, M., Yamamoto, K. and Fukuda, N. (2000) Effect of Momoridica charantia powder on serum glucose levels and various lipid parameters in rat fed with cholesterol-free cholesterol-enriched diets. Journal of Ethnopharmacology, 72, 331-336. doi:10.1016/S0378-8741(00)00259-2

[6] Han, C., Hui, Q. and Wang, Y. (2008) Hypoglycaemic activity of saponin fraction extracted from Momoridica charantia in PEG/salt aqueous two-phase systems. Natural Product Research, 22, 1112-1119. doi:10.1080/14786410802079675

[7] Tan, M-J., Ye, J-M., Turner, N., Hohnen-Behrens, C., Ke, 
C-C., Tang, C-P., Chen, T., Weiss, H-C., Gessing, E-R., Rowland, A., James, D.E. and Ye, Y. (2008) Antidiabetic activities of Triterpenoids isolated from bitter melon associated with activation of the AMPK pathway. Chemistry and Biology, 15, 263-273.

doi:10.1016/j.chembiol.2008.01.013

[8] Shih, C.-C., Lin, C.-H., Lin, W.-L. and Wu, J.-B. (2009) Momoridica charantia extract on insuline resistance and the skeletal muscle GLUT4 protein in fructose-fed rats. Journal of Ethnopharmacology, 123, 82-90. doi:10.1016/i.jep.2009.02.039

[9] Kumar, R., Balaji, S., Uma, T.S. and Sehgal, P.K. (2009) Fruit extracts of Momoridica charantia potentiate glucose uptake and up-regulate Glut-4, PPAR $\gamma$ and P13K. Journal of Ethnopharmacology, 126, 533-537. doi:10.1016/j.jep.2009.08.048

[10] Guevara, A.P., Lim-Slianco, C., Dayrit, F. and Finch, P. (1990) Antimutagens from Momoridica charantia. Mutation Research, 230, 121-126. doi:10.1016/0027-5107(90)90050-E

[11] Lee-Huang, S., Huang, P.L, Huang, P.L. Bourinbaiar, A.S., Chen, H.C. and Kung, H.F. (1995) Inhibition of the integrase of human immunodeficiency virus (HIV) type1 by anti HIV plant proteins MAP30, and GAP31. Proceedings of National Academy of Science USA, 92, 88188822.

[12] Reaven, G.M. and Laws, A. (1994) Insulin resistance, compensatory hyperinsulinemia, and coronary heart disease. Diabetologia, 37, 948-952. doi:10.1007/BF00400953

[13] Manabe, M., Takenaka, R., Nakasa, T. and Okinaka, O. (2003) Induction of anti-inflammatory response by dietary Momoridica charantia. L. (Bitter gourd). Bioscience, Biotechnology, and Biochemistry, 67, 2512-2517. doi:10.1271/bbb.67.2512

[14] Cefalu, W.T., Ye, J. and Wang, Z.Q. (2008) Efficacy of dietary supplementation with botanicals on carbohydrate metabolism in humans. Endocrine, Metabolic and Immune Disorders-Drug Target, 8, 78-81.

[15] Nerurkar, P.V., Lee, Y.K., Linden, E.H., Lim, S., Pearson, L., Frank, J. and Nerurkar, V.R. (2006) Lipid lowering effect of Momoridica charantia (Bitter melon) in HIV1-protease inhibitor-treated human hepatoma cells, HepG2. British Journal of Pharmacology, 148, 115-1164. doi:10.1038/sj.bjp.0706821

[16] Singh, A., Singh, S.P. and Bamezai, R. (1998) Momoridica charantia (Bitter gourd) peel, pulp, seed and whole fruit extract inhibits mouse skin papillomagnesis. Toxicology Letters, 94, 37-46. doi:10.1016/S0378-4274(97)00099-4
[17] Munsur, M.A.Z., Haque, M.S., Nasiruddin, K.M. and Hossain, M.S. (2009) In vitro propagation of bitter gourd (Momoridica charantia L.) from nodal and root segments. Plant Tissue Culture and Biotechnology, 19, 45-52.

[18] Malek, M.A., Miah, B.M.A. and Al-Amin, M. (2007) In vitro regeneration in pointed gourd. Bangladesh Journal of Agricultural Research, 32, 461-471.

[19] Tang, Y., Liu, J., Li, X.-M., Liu, B. and Li, H.-X. (2011) The influence of endogenous hormones on the formation of buds from stems of bitter melon (Momoridica charantia L.). African Journal of Biotechnology, 10, 5856-5860.

[20] Liao, C.T. and Lin, C.H. (1996) Photosynthetic responses of grafted bitter melon seedlings to flood stress. Environmental and Experimental Botany, 36, 167-172. doi:10.1016/0098-8472(96)01009-X

[21] Wang, S., Tang, L. and Chen, F. (2001) In vitro flowering of bitter melon. Plant Cell Reproduction, 20, 393-397. doi:10.1007/s002990100351

[22] Rugini, E., Jacoboni, A. and Luppino, M. (1993) Role of basal shoot darkening and exogenous putrescine treatments on invitro rooting and on endogenous polyamine changes in difficult-to-root woody species. Scientia Horticulturae, 53, 63-72. doi:10.1016/0304-4238(93)90138-G

[23] Hausman, J.-F., Kevers, C. and Gaspar, T. (1994) Involvement of putrescine in the inductive rooting phase of popular shoots raised in vitro. Physiologia Plantarum, 92, 201-206. doi:10.1111/j.1399-3054.1994.tb05327.x

[24] Malik, N.S.A. and Bradford, J.M. 2007. Different flowerinducing conditions elicit different responses for free polyamine levels in olive Olea europaea leaves. Journal of Japanese Society of Horticulture Science, 76, 205-209. doi: 10.2503/jishs. 76.205

[25] Thimann, K.V. (1977) Hormone action in the whole life of plants. The University of Massachusetts Press, Amherst.

[26] Rey, M., Diaz-Sala, C. and Rodriguez, R. (1994) Exogenous polyamines improve rooting of hazel microshoots. Plant Cell, Tissue and Organ Culture, 36, 303-308. doi:10.1007/BF00046087

[27] Jarvis, B.C., Shannon, P.R.M. and Yasmin, S. (1983) Involvement of polyamines with adventitious root development in stem cuttings of mung bean. Plant and Cell Physiology, 24, 677-683.

[28] Nag, S., Saha, K. and Choudhuri, M.A. (2001) Role of auxin and polyamines in adventitious root formation in relation to changes in compounds involved in rooting. Journal of Plant Growth Regulation, 20, 182-194. doi:10.1007/s003440010016 